\title{
PRODUCTION DE JUVENILES DE SAUMON ATLANTIQUE (SALMO SALAR L.) \\ DANS QUATRE AFFLUENTS DU SCORFF, RIVIERE DE BRETAGNE-SUD
}

\author{
par J. L. BAGLINIERE 1
}

De 1973 à 1975 des alevins de Saumon atlantique (Salmo salar L.) ont été déversés en fin de résorption de vésicule dans quatre affluents du Scorff, rivière de Bretagne-Sud. Dans le même temps, les milieux d'alevinage ont été aménagés : création de profonds et enlèvement des populations de truites et d'anguilles.

Les densités et les tailles moyennes automnales des saumons ont varié selon les ruisseaux et les années de 1,3 à 16 ind./100 $\mathrm{m}^{2}$ pour la classe d'âge $0^{+}$(52 à $107 \mathrm{~mm}$ ) et de 0,6 à 6,9 ind. $/ 100 \mathrm{~m}^{2}$ pour celle des $1^{+}(114$ à $150 \mathrm{~mm})$. La production en migrants (observée sur une année et un ruisseau) reste faible et est composée de juvéniles parrs et smolts âgés de 1 et 2 ans.

La densité d'alevinage en relation avec l'habitat, la suppression des populations de truites et d'anguilles et l'aménagement du milieu influent sur la densité et la survie automnales des $0^{+}$. Les actions individuelle et synergique de ces facteurs sur la production sont discutées.

\section{Production of juveniles of Atlantic salmon (Salmo salar L.) in four tributaries of the Scorff, a river in south Brittanny.}

From 1973 to 1975, Atlantic salmon alevins were stocked at the end of the volk-sac resorption in four Scorff tributaries, a South-Brittany river. At the same time, the stocked brooks have been managed by creating pools and removing trout and eel populations.

Salmon density and mean autumnal size have ranged according to the tributaries and years from 1,3 to 16 ind. $/ 100 \mathrm{~m}^{2}$ for the $0^{+}$age class $(52$ to $107 \mathrm{~mm})$ and from 0,6 to 6,9 ind./100 $\mathrm{m}^{2}$ for the $1^{+}$age class $(114$ à $150 \mathrm{~mm}$ ). Production in juvenile migrants (observed over a year in one tributary) is low and is made up of one and two years old parrs and smolts.

Stocking density in rclation with the habitat, removal of trout and eel populations and habitat improvment have a influence on the salmon $0^{+}$density and autumnal survival. The individual and synergistic actions of these factors on the production are discussed.

\section{1. - INTRODUCTION}

L'étude des populations pisciaires du bassin du Scorff a montré une forte présence de juvéniles de truites et une quasi-absence de production en saumon sur les affluents (Baglinière 1979).

1. I.N.R.A., Laboratoire d'Ecologie hydrobiologique, E.N.S.A., 65, rue de SaintBrieuc, 35042 Rennes Cedex. 
A partir de 1973 il a été décidé d'expérimenter une augmentation de la production en saumon atlantique : des alevins de saumon ont été déversés en fin de résorption de vésicule dans quatre affluents du Scorff ayant subi certains aménagements.

Cet article expose les résultats obtenus en les comparant à des expériences similaires réalisées en Bretagne sur l'Elorn (Prouzet et al., 1977) et dans d'autres pays (Mc Crimmon 1954, Mills 1964 et 1969, Saunders et Gee 1964, Elson 1975).

\section{2. - PRESENTATION DU BASSIN DU SCORFF}

Le bassin du Scorff a été décrit dans un premier article (Baglinière 1979). Rappelons que ce petit fleuve côtier est localisé en BretagneSud. Son cours, long de $75 \mathrm{~km}$, a un bassin versant de $480 \mathrm{~km}=$. Sa pente moyenne est de $3,6 \%$ et son débit moyen est de $5 \mathrm{~m}^{3}$ par seconde.

Les quatre affluents étudiés sont situés sur la rive droite de la partie aval du bassin : ce sont les ruisseaux de Penlan, Kernec, Kerlégan et Kerloas (fig. 1). Leurs longueurs sont comprises entre 4 et 6 kilomètres pour une largeur moyenne de 1,61 à 2,24 mètres. Leur pente varie selon les secteurs entre 2 et 16 pour mille. Les ruisseaux de Kernec et de Kerlégan, les parties amont des ruisseaux de Penlan et de Kerloas ont les plus fortes pentes (en moyenne supérieures à $6 \%$ ) et sont constituées principalement d'une succession de radiers et profonds comme celle décrite par Mundie (1974) (fig. 1). Les parties avals des ruisseaux de Penlan et Kerloas sont des milieux à pente faible (inférieure à $4 \%$ ) et à eaux calmes (profonds supérieurs à $1 \mathrm{~m}$, fig. 1).

\section{3. - TECHNIQUES D'ETUDE}

\subsection{Aménagement et enlèvement des populations}

Dans le ruisseau de Kerloas de petits barrages (création de zones profondes) ont été construits sur des secteurs alternant avec d'autres non aménagés sur une distance de 300 mètres (fig. 1).

La population d'anguilles a toujours été enlevée avant chaque déversement et lors de chaque contrôle, sauf dans le ruisseau de Penlan en 1974 et sur un secteur du ruisseau de Kerlégan en 1975. La population de truites a été enlevée en octobre, novembre 1972, février 1973 sur tous les ruisseaux et en avril 1974 sur ceux de Kernec et Kcrloas. Elle a également été enlevée trimestriellement sur un secteur du ruisseau 
Fig. 1. - Caractéristiques des milieux d'étude et types d'aménagement réalisés sur quatre affluents du Scoff lors de déversement d'alevins de saumon atlantique de 1973 à 1975.
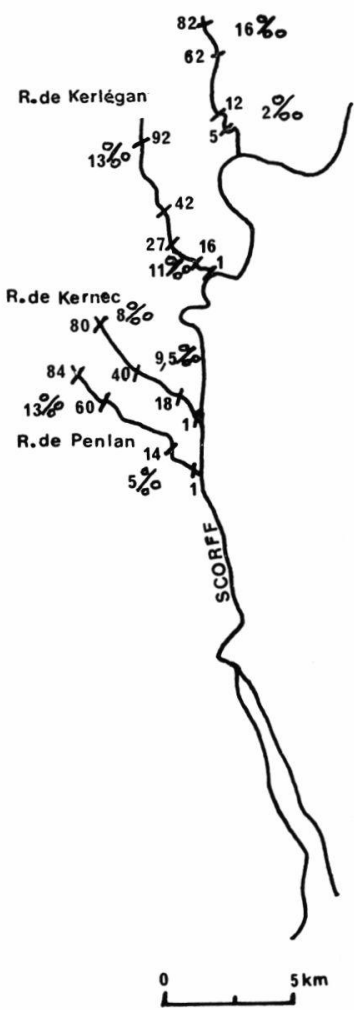

\begin{tabular}{|c|c|c|c|c|c|}
\hline Année & $\begin{array}{l}\text { Zone } \\
\text { alevinée }\end{array}$ & $\begin{array}{l}\text { Nombre } \\
\text { d'alevins } \\
\text { et densité } \\
\text { (ind./m2) }\end{array}$ & $\begin{array}{l}\text { Date } \\
\text { d'alevi- } \\
\text { nage }\end{array}$ & $\begin{array}{l}\text { Aménagement } \\
\text { et enlèvement } \\
\text { de poissons }\end{array}$ & Mois d'izventaire \\
\hline 1973 & $5: 12$ & $\begin{array}{c}14000 \\
10\end{array}$ & $\begin{array}{l}\text { RuISSEAU } \\
7 \text { avril }\end{array}$ & $\begin{array}{l}\text { DE KERLOAS } \\
\text { construction de barrages } \\
\text { enlèvement des truites et } \\
\text { des anguilles }\end{array}$ & $\begin{array}{l}\text { juillet } \\
\text { octobre }\end{array}$ \\
\hline 1974 & $62-82$ & 9800 & 7 avril & $\begin{array}{l}\text { enlèvement des truites ef } \\
\text { des anguilles }\end{array}$ & $\begin{array}{l}\text { janvier, avril } \\
\text { juillet, octobre }\end{array}$ \\
\hline 1975 & - & 0 & - & - & avril \\
\hline 1973 & $1-16$ & $\begin{array}{l}16000 \\
10\end{array}$ & $\begin{array}{l}\text { RUISSEAU } \\
7 \text { avril }\end{array}$ & $\begin{array}{l}\text { DE KERLEGAN } \\
\text { enlèvement des truites et } \\
\text { des anguilles }\end{array}$ & $\begin{array}{l}\text { juillet } \\
\text { octobre }\end{array}$ \\
\hline 1974 & $16-92$ & 41800 & $\begin{array}{l}7 \text { au } \\
10 \text { avril }\end{array}$ & $\begin{array}{l}\text { enlèvement des truites et } \\
\text { des anguilles }\end{array}$ & $\begin{array}{l}\text { janvier, avril } \\
\text { juillet, octobre }\end{array}$ \\
\hline 1975 & $27-42$ & $\begin{array}{c}10000 \\
5\end{array}$ & 27 mars & $\begin{array}{l}\text { enlèvement sectoriel des } \\
\text { truites et des anguilles }\end{array}$ & $\begin{array}{l}\text { juillet } \\
\text { octobre }\end{array}$ \\
\hline 1973 & $1-18$ & $\begin{aligned} 13300 \\
10\end{aligned}$ & $\begin{array}{l}\text { RuISSEAU } \\
5 \text { et } \\
7 \text { avril }\end{array}$ & $\begin{array}{l}\text { J DE KERNEC } \\
\text { enlèvement des truites et } \\
\text { des anguilles }\end{array}$ & octobre \\
\hline 1974 & $18-80$ & 26200 & 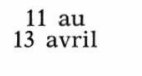 & $\begin{array}{l}\text { enlèvement des truites et } \\
\text { des anguilles }\end{array}$ & $\begin{array}{c}\text { avril, mai, juin } \\
\text { juillet, septembre } \\
\text { octobre, décembre }\end{array}$ \\
\hline 1975 & $1-40$ & $\begin{array}{c}18000 \\
5\end{array}$ & 27 mars & enlèvement des anguilles & juillet, octobre \\
\hline 1973 & $1-14$ & 16300 & $\begin{array}{l}\text { RuISSEAU } \\
7 \text { avril }\end{array}$ & $\begin{array}{l}\text { DE PENLan } \\
\text { enlèvement des truites et } \\
\text { des anguilles }\end{array}$ & octobre \\
\hline 1974 & $60-84$ & 8000 & 12 avril & & octobre \\
\hline
\end{tabular}


de Kerlégan (fig. 1). Signalons qu'après les enlèvements de population en 1972, des grilles ont été posées en amont et en aval des secteurs à aleviner.

\subsection{Alevinage}

De 1973 à 1975, 173400 alevins de saumon de souche écossaise sont déversés en fin de résorption de vésicule. Leur répartition par ruisseau et leur densité (5 à 10 ind./ $\mathrm{m}^{2}$ ) ont varié selon les années (fig. 1). L'alevinage a été effectué entre le 24 mars et le 13 avril sur une distance s'échelonnant entre 350 et 3800 mètres (fig. 1).

\subsection{Obtention des données}

Des inventaires par pêche électrique ont été effectués dans les secteurs alevinés et non alevinés à intervalles variables (1 mois à 12 mois) (fig. 1). Les densités (ind./100 $\mathrm{m}^{2}$ ) sont estimées par la méthode de Seber et Lecren. Il sera plutôt fait référence à la densité de la classe des $0^{+}$puisqu'une partie de la population de juvéniles peut migrer vers la mer dès l'âge d'un an (Baglinière 1976; Fontenelle et al en prép.). La croissance est mesurée par la variation de la longueur moyenne à la fourche.

Afin d'estimer l'impact de l'enlèvement des populations de truites et d'anguilles sur la densité de saumon $0^{+}$, les données obtenues sur lesquatre ruisseaux et durant les trois années ont été regroupées et traitées de la manière suivante:

- pour l'Anguille, des classes d'intervalle de temps séparant deux pêches d'enlèvement (échelle de 2 mois) ont été définies. Elles sont au nombre de 8 allant de 2 à 16 mois. Pour chacune de ces classes, il a été calculé une variation moyenne de densité exprimée en pourcentage à partir des valeurs trouvées aux pêches précédentes.

- pour la Truite, un facteur de multiplication annuelle de population a été utilisé, que l'enlèvement ait été réalisé au printemps ou en automne.

En plus des inventaires, les déplacements des juvéniles de saumons ont été étudiés en 1975 par marquage sur le ruisseau de Kerlégan (trois secteurs de $300 \mathrm{~m}$ ) et par contrôle à l'aide d'un piège de descente situé au confluent du ruisseau de Kernec avec la rivière.

\section{4. - RESULTATS}

\subsection{Densité et survie dans les secteurs alevinés}

La densité moyenne automnale (établie sur trois ans) en Saumon est de 5,9 ind./100 $\mathrm{m}^{2}$ regroupant les deux classes d'âge : 4,6 ind./ 
$100 \mathrm{~m}^{2}$ (de 1,3 à 16 ind. $/ 100 \mathrm{~m}^{2}$ ) pour les $0^{+}$et 1,3 ind. $/ 100 \mathrm{~m}^{2}$ (de 0,6 à 6,9 ind. $/ 100 \mathrm{~m}^{+}$) pour les $1^{+}$.

A l'exception du ruisseau de Kerloas, elles sont les plus élevées en 1973 (fig. 2). En moyenne les plus fortes densités sont observées sur les ruisseaux de Kernec (jusqu'à 24 ind./100 $\mathrm{m}^{2}$ ) et de Kerlégan (fig. 2).

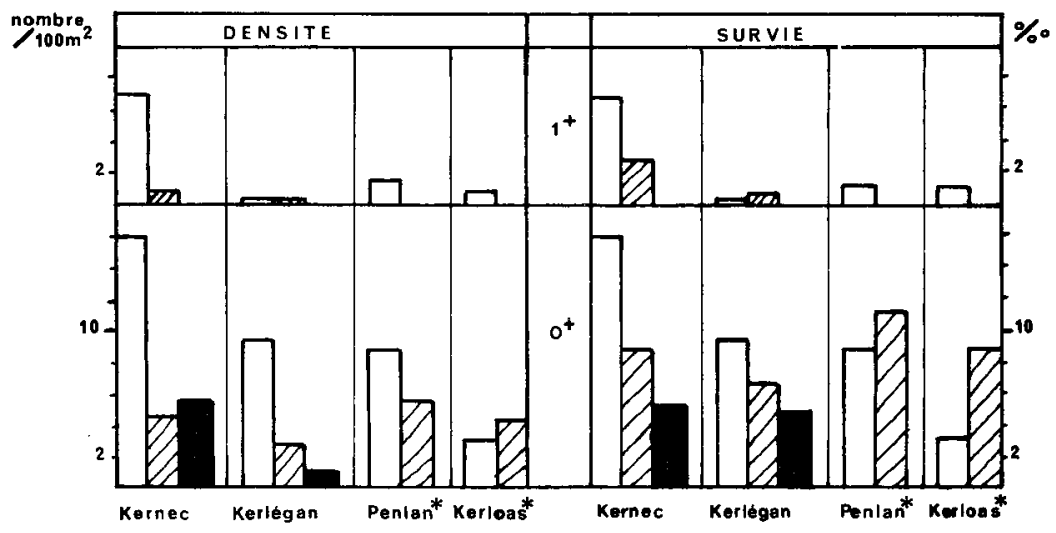

Fig. 2. - Densité (ind. $/ 100 \mathrm{~m}^{2}$ ) et survie $\left(\%{ }_{0}\right.$ ) automnales pour les classes d'âge $0^{+}$et $1^{+}$de Saumon atlantique déversés sur quatre affluents du Scorff de 1973 à 1975. (blanc) 1973, (hachuré) 1974, (noir) 1975.

* Aucun alevinage n'est réalisé sur les ruisseaux de Penlan et Kerloas en 1975.

La survie depuis le stade alevin jusqu'au stade $0^{+}$varie de $0,26 \%$ à $1,6 \%$. Les plus élevées sont constatées sur les ruisseaux de Kerlégan et de Kernec (sur un secteur de $2,5 \%$ ) pour une densité initiale de 1000 alevins par $100 \mathrm{~m}^{2}$ (fig. 2). Pour ceux de Penlan et Kerloas les meilleures survies sont observées en 1974 pour une densité initiale de 500 alevins par $100 \mathrm{~m}^{2}$ (fig. 2). Pour cette valeur, la survie est par contre très faible sur les ruisseaux de Kernec et Kerlégan en 1975 (fig. 2). Le taux de survie jusqu'à l'âge d'un an a été estimé à $1,1 \%$ sur l'ensemble du ruisseau de Kernec en avril 1974.

\subsection{Déplacements à l'intérieur des ruisseaux}

Entre les trois secteurs alevinés du ruisseau de Kerlégan, on constate des déplacements mais seulement pour les juvéniles de saumon $1^{+}$. Cette population de saumons migrants représente en moyenne $27,5 \%$ et $25 \%$ de la population totale recensée respectivement en mars et juillet 1975 .

La présence de juvéniles de saumons en amont et en aval des secteurs alevinés est constatée chaque année à différentes périodes. Les densités totales oscillent entre 0,3 et 7,5 individus par $100 \mathrm{~m}^{2}$. Les 
plus fortes sont observées en aval pour la classe d'âge $0^{+}$sur les ruisseaux de Penlan en 1973 (octobre : 5,9 ind./100 $\mathrm{m}^{2}$ ) et de Kernec en 1974 (juillet : 7,5 ind./100 $\mathrm{m}^{2}$ et octobre : 6,2 ind./100 $\mathrm{m}^{2}$ ).

Les jeunes saumons se déplacent sur des longueurs (au moins égales ou supérieures) allant de 0 à $3,6 \mathrm{~km}$. Les déplacements les plus longs sont observés en aval (3,6 km contre $2,5 \mathrm{~km}$ en amont), pour la classe d'âge $1^{+}$(avril, juillet ou octobre) et sur le ruisseau de Kerloas où la densité de saumons diminue avec l'importance du déplacement (fig. 3). Pour les juvéniles $0^{+}$, les plus longs déplacements sont observés en octobre en aval ( $2 \mathrm{~km}$ contre $0,1 \mathrm{~km}$ en amont). Signalons qu'en 1975 sur le ruisseau de Kernec trois alevins son récupérés dans le piège de descente dans les deux semaines suivant le déversement.

La longueur des déplacements ne peut être reliée à la densité d'alevinage en raison du changement annuel des zones alevinées.

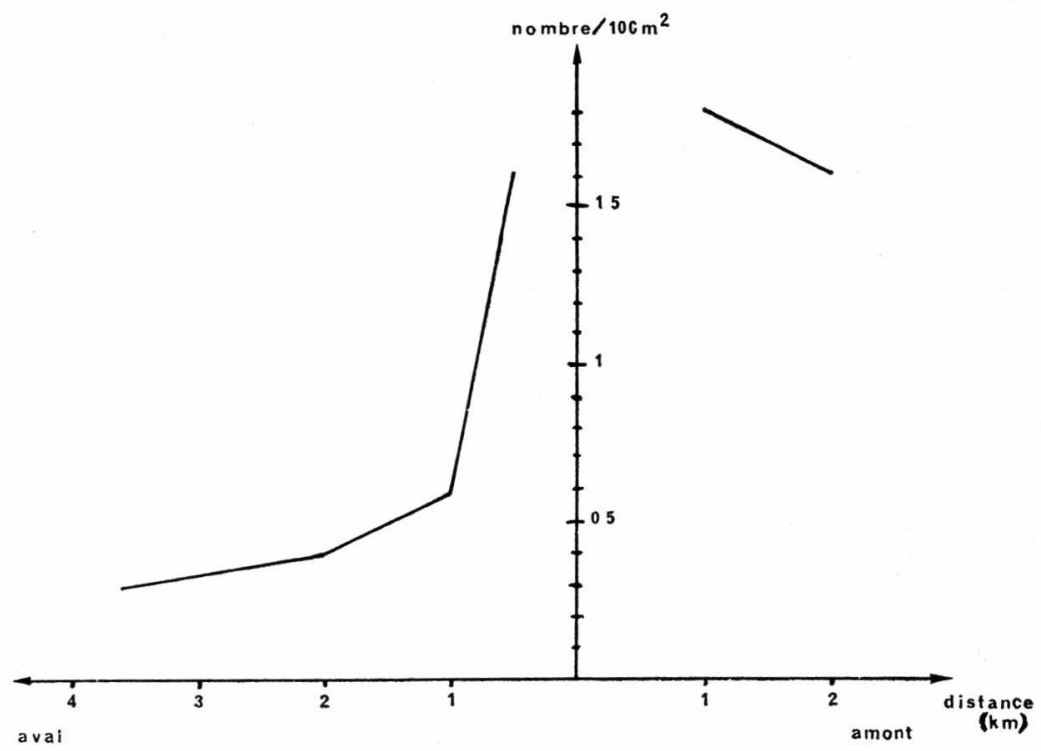

Fig. 3. - Densité de saumon $1^{+}$(ind./100 $\mathrm{m}^{2}$ ) et longueur du déplacement par rapport aux secteurs alevinés sur le ruisseau de Kerloas en avril 1974 et 1975.

\subsection{Variations spatio-temporelles de la densité}

Sur l'ensemble des secteurs inventoriés au cours de l'étude, il apparaît :

- sur le ruisseau de Kernec, une forte diminution de la classe d'âge $0^{+}$de mai à juillet 1974 dans les secteurs alevinés au profit d'un secteur non aleviné de la partie aval (fig. 4 A). En 1975, un gradient 
croissant de densité d'amont en aval au mois d'octobre par rapport à celui de juillet (fig. $4 \mathrm{~B}$ ).

- sur les trois ruisseaux de Kernec, Kerloas, d'abord une stabilité ou une diminution des effectifs de saumons $0^{+}$de juillet à septembreoctobre, puis une forte diminution de cette classe d'âge de novembre à janvier ou avril et enfin une baisse du nombre de saumons $1^{+}$d'avril à juin (fig. $4 \mathrm{~A}$ et fig. 5).

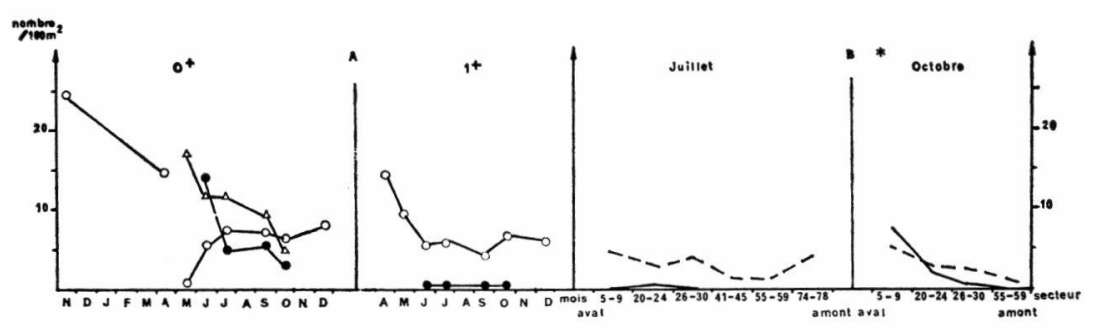

Fig. 4. - Evolution de la densité de population (ind./100 $\mathrm{m}^{2}$ ) de Saumon sur le ruisseau de Kernec.

(A) de novembre 1973 à décembre 1974 pour les classes d'âge $0^{+}$et $1^{+}$sur trois secteurs : 5-9 non aleviné (blancs), 41-45 aleviné (noirs), 55-59 aleviné (triangles). (B) d'aval en amont aux mois de juillet et d'octobre 1975 pour les classes d'âge $0^{+}$ et $1^{+}$.......

* Les secteur 41-45 et 55-59 n'ont pas été inventoriés en octobre.

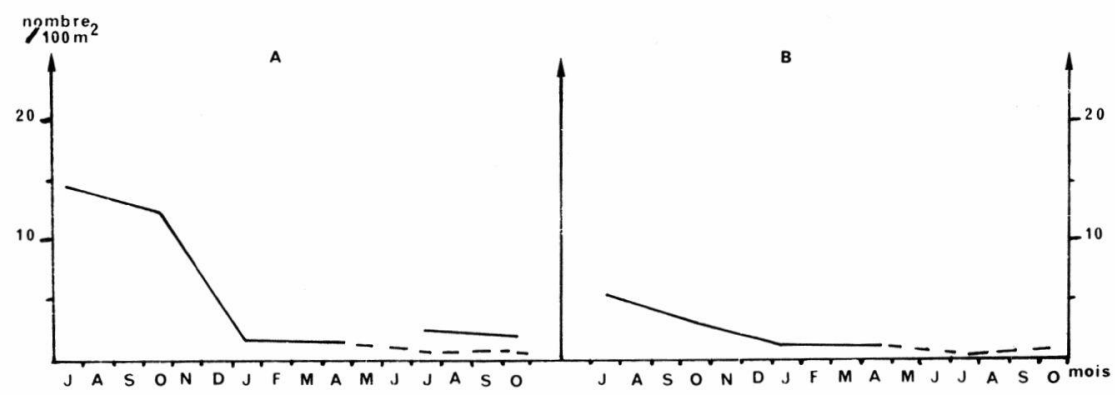

Fic. 5. - Evolution de la densité de population (ind./100 $\mathrm{m}^{2}$ ) de Saumon âgé de $0^{+} \longrightarrow$ et de $1^{+} \ldots .$. ... de juillet 1973 à octobre 1974.

(A) sur le ruisseau de Kerlégan.

(B) sur le ruisseau de Kerloas.

\subsection{Croissance}

La taille automnale varie de 52 à $107 \mathrm{~mm}$ pour la classe d'âge $0^{+}$ (moyenne 73,8 mm) et de 114 à $150 \mathrm{~mm}$ pour celle des $1^{+}$an (moyenne $126,6 \mathrm{~mm})$. La croissance est toujours plus faible sur le ruisseau de Penlan alors qu'elle semble du même ordre sur les trois autres (Tableau I). De plus des différences peuvent exister entre les secteurs d'un même ruisseau. Sur celui de Kernec, la taille des saumons $0^{+}$ 
dans la partie aval est significativement plus élevée $(85,7 \mathrm{~mm}$ en octobre 1974) que celle observée dans deux secteur amont $(73,3$ et $77,7 \mathrm{~mm}$ ). C'est également le cas sur le ruisseau de Kerlégan. Seule la comparaison de mêmes secteurs fait apparaître une meilleure croissance en 1974 qu'en 1973 sur ces deux ruisseaux.

Tableau I. - Taille automnale (en $\mathrm{mm})$ des juvéniles de saumon atlantique $\left(0^{+}\right.$et $\left.1^{+}\right)$déversés sur quatre affluents du Scorff de 1972 à 1976.

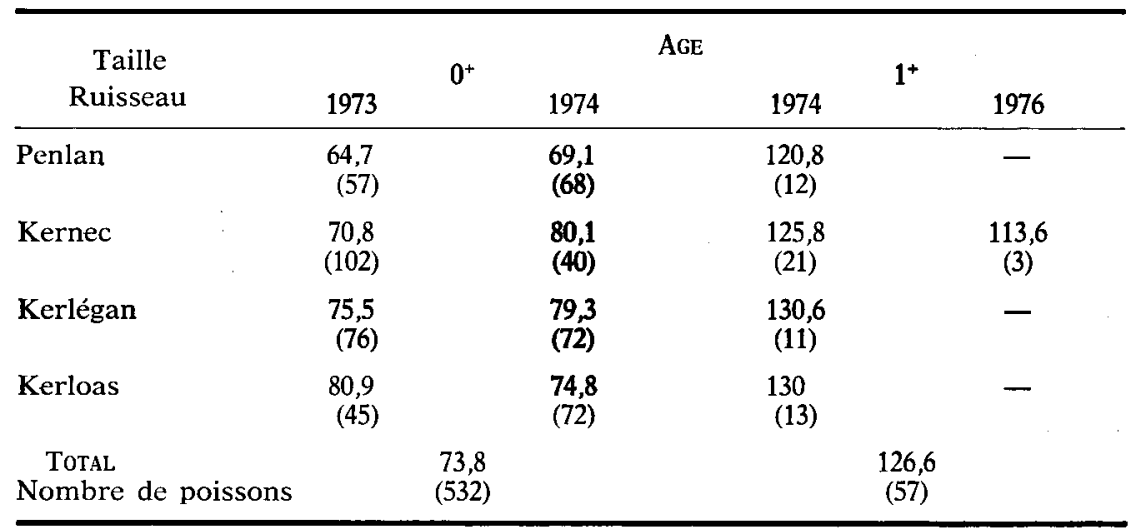

\subsection{Influence de l'aménagement et de l'enlèvement des populations}

\subsubsection{CONSTRUCTION DE BARRAGES}

Dans le ruisseau de Kerloas, la densité de population de saumon du secteur aménagé est toujours supérieure à celle du secteur sans barrage (fig. 6). Le rapport de densité dans la partie aménagée à celle non aménagée varie au cours de l'année. Pour la classe d'âge $0^{+}$il passe de 2,8 en novembre à 3,6 en janvier et pour celle des $1^{+}$de 1,3 en avril à 7 en juillet.

\subsubsection{ENLÈVEMENT DES POPULATIONS DE TRUITES ET D'ANGUILles}

- Ensemble des ruisseaux

La densité en saumon diminue lorsque la population d'anguilles augmente, c'est-à-dire lorsque l'intervalle de temps séparant deux pêches d'enlèvement est supérieur à 6 mois (fig. 7). Par contre une diminution de la population d'anguilles (pêches d'enlèvement fréquentes) influe peu sur la densité de jeunes saumons.

L'enlèvement de la population de truites semble aussi influer sur la densité de saumons $0^{+}$. Quand l'opération est effectuée en automne (ou l'hiver), le nombre de truites présentes un an après est en moyenne multiplié par 1,6 (de 1,2 à 1,94) et plus la densité de truites s'est élevée, plus celle des saumons $0^{+}$observée à l'automne est forte (fig. 8). 
Après une telle opération, la densité moyenne de saumons atteint 10,94 ind./ $100 \mathrm{~m}^{2}$ contre 5,5 ind./100 $\mathrm{m}^{2}$ sans enlèvement des truites. Lorsque cette opération est réalisée au printemps (avril), le nombre de truites présentes un an après est en moyenne multiplié par $1,2(0,8$ à 1,8$)$ et la densité automnale de saumons augmente beaucoup moins (fig. 8). Les plus hautes valeurs sont observées pour un facteur de multiplication compris entre 1,1 et 1,3 (fig. 8).

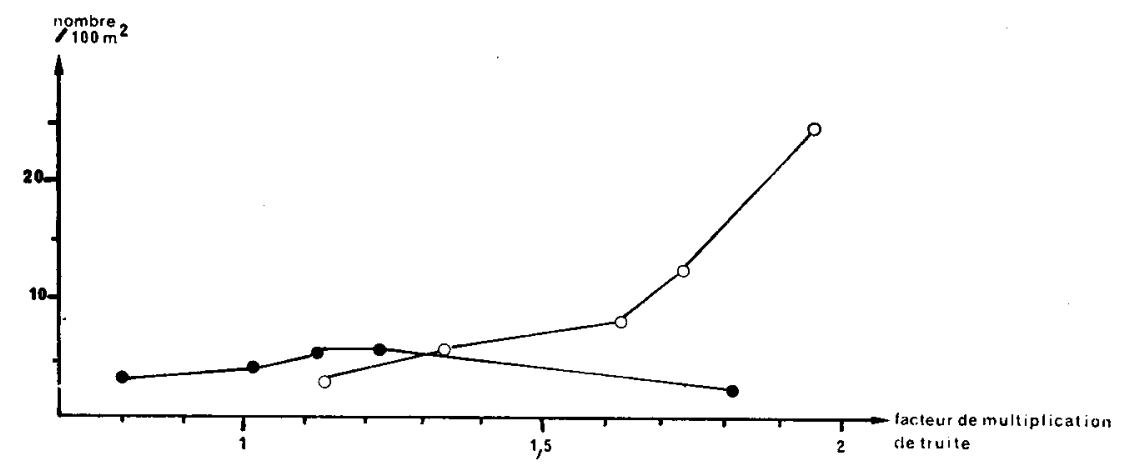

Fig. 8. - Evolution de la densité de saumon (ind./100 $\mathrm{m}^{2}$ ) en relation avec le facteur de multiplication de la population de Truite après enlèvement en octobre (cercle blanc) et en avril (cercle noir) sur les quatre affluents.

\section{- Ruisseau de Kerlégan}

La densité en saumon apparaît supérieure dans le secteur où les

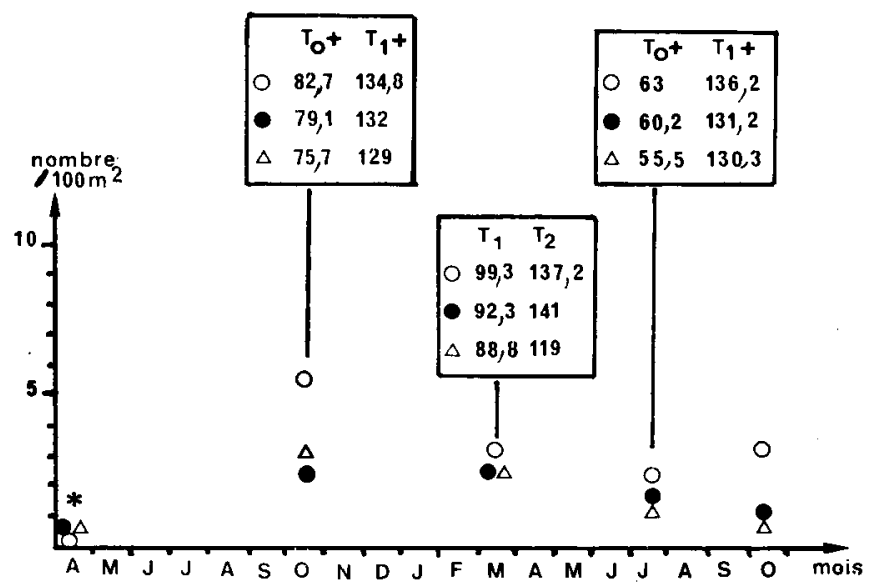

Fig. 9. - Evolution de la densité de saumon (ind./100 $\mathrm{m}^{2}$ ) d'avril 1974 à octobre 1975 dans trois secteurs du ruisseau de Kerlégan.

(cercle blanc) Truite et anguille enlevées; (cercle noir) anguille enlevée; (triangle) Aucune espèce enlevée.

$\mathrm{T} 0^{+}, 1,1^{+}$ou 2 : Taille moyenne (longueur fourche en $\mathrm{mm}$ ) des saumons âgés de $0^{+}, 1,1^{+}$ou 2 ans.

* L'enlèvement partiel des espèces n'a commencé qu'au mois d'avril 1974. 
truites et les anguilles sont enlevées tous les trois mois mais il ne semble pas y avoir une augmentation significative de la taille du jeune saumon lorsque l'on enlève ces deux espèces (fig. 9).

\subsection{Migration vers la rivière et rendement en migrants}

Du 11 janvier au 2 mai 1975, 79 jeunes saumons sont capturés dans le piège de descente sur le ruisseau de Kernec, $81 \%$ d'entre eux étant smolt ou présmolt.

\subsubsection{TAILLE ET AGE}
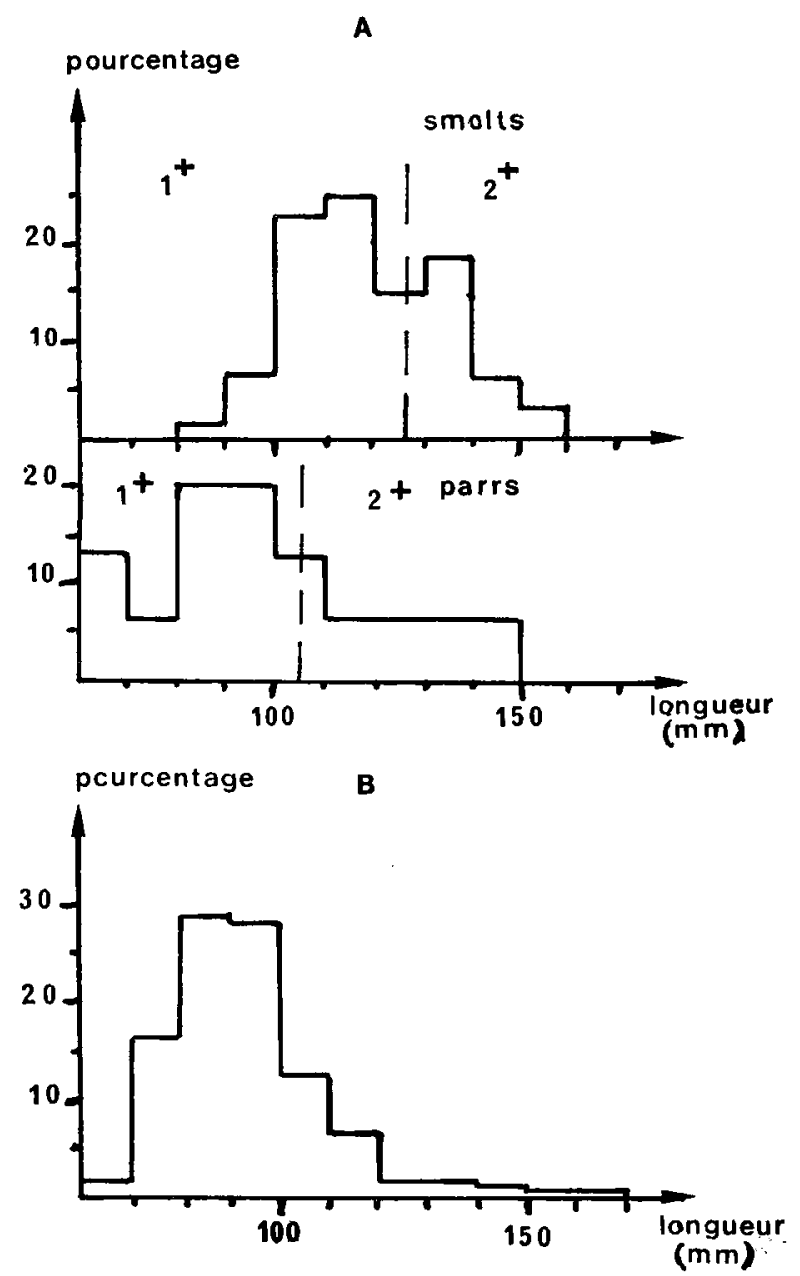

Fig. 10. - Histogramme de fréquence de taille (\%).

(A) des saumons parrs et smolts migrants sur le ruisseaux de Kernec en 1975.

(B) des saumons parrs sédentaires capturés aux mois de mars et avril 1974 et 1975 sur tous les ruisseaux. 
La longueur des parrs s'échelonne de 63 à $141 \mathrm{~mm}$ (moyenne $98 \mathrm{~mm}$ ) et celle des smolts de 87 à $152 \mathrm{~mm}$ (moyenne $116 \mathrm{~mm}$ ). Les classes de taille les plus représentées se situent entre 80 et $100 \mathrm{~mm}$ pour les parrs et entre 100 et $120 \mathrm{~mm}$ pour les smolts ( $f i g .10 \mathrm{~A}$ ).

Les parrs migrants sur le ruisseau de Kernec ont des tailles semblables à celles des parrs sédentaires capturés sur tous les ruisseaux en mars et avril 1974 et 1975 (fig. $10 \mathrm{~B}$ ).

Les juvéniles migrants sont âgés de un et deux ans, le recouvrement des deux classes d'âge étant important. Une estimation (méthode de Harding) donne une répartition en $60 \%$ de un an et $40 \%$ de deux ans.

\subsubsection{RYTHME}

$84,8 \%$ des captures sont réalisées durant les mois de mars et avril. Cela correspond à la période de dévalaison des smolts ( $81 \%$ des migrants) s'étendant de mars à mai avec un maximum en avril $(61,5 \%$ des smolts) (fig. 11). Par contre les parrs migrent plus tôt, de janvier à mars (fig. 11). Il faut toutefois noter la présence de smolts en janvier, fait également constaté en 1974 sur l'aval du ruisseau de Kerlégan.

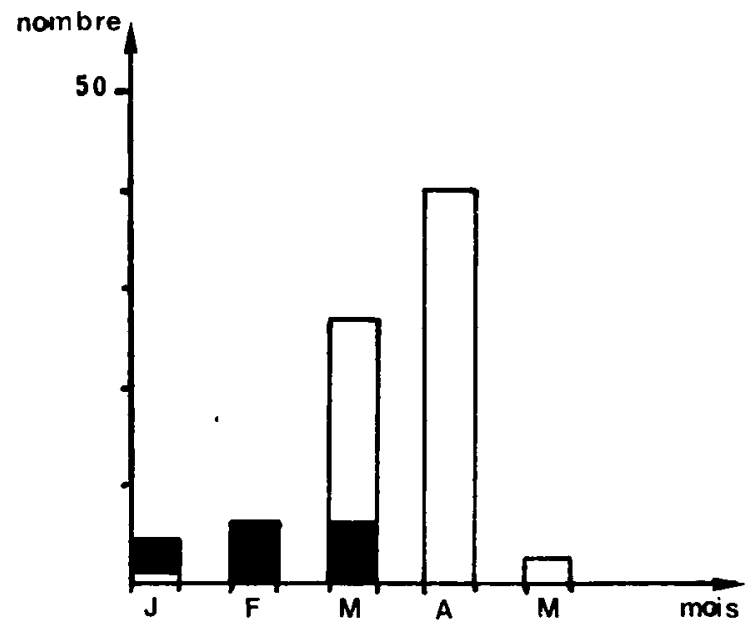

Fic. 11. - Nombre mensuel de jeunes saumons (smolts ou présmolt : carré blanc ; parr: carré noir) capturés lors de leur dévalaison sur le ruisseau de Kernec de janvier à mai 1975 .

\subsubsection{RENDEMENT EN MIGRANTS}

Le rendement en migrants est calculé avec les données d'une seule année. A partir du nombre d'alevins déversés sa valeur est de 3,8\% pour une densité estimée de 1,4 migrants par $100 \mathrm{~m}^{2}$ sur tout le ruisseau. En considérant, pour la migration 1974, une même répartition des deux classes d'âge, le rendement en migrants $2^{+}$est de $6 \%$ et a une valeur double de celui en migrants $1^{+}$. 


\section{5. - DISCUSSION}

\subsection{Densité et survie}

La densité totale des deux classes d'âge de saumons déversés dans les quatre affluents est supérieure à celle du saumon naturel présent dans le cours principal du Scorff de 1972 à 1976 (Baglinière 1979). Toutefois le rapport des deux classes d'âge $\left(1^{+} / 0^{+}\right)$reste sensiblement le même (25\% dans la rivière et $28 \%$ dans les affluents).

Les résultats obtenus dans cette étude sont du même ordre que ceux observés sur 1'Elorn (Bretagne-Nord) (Prouzet et al. 1977) mais diffèrent de ceux constatés dans d'autres expériences similaires (Tableau II).

Ces différences et les variations annuelles observées peuvent s'expliquer au travers de nombreux facteurs. Ceux-ci sont plus particulièrement analysés pour la classe d'âge $0^{*}$ compte tenu d'une mortalité nettement plus faible après ce stade (Mc Crimmon 1954, Mills 1964 et 1969, Egglishaw et Shackley 1977) et d'une dévalaison de $60 \%$ de jeunes saumons à un an.

\section{- le stade de déversement}

Le meilleur stade de déversement varie selon les auteurs: pour Sedgewick (1970) c'est le stade œuf. Mais sur l'Elorn, Prouzet et al. (1977) constatent une plus faible réussite avec un déversement effectué au stade œuf qu'avec des alevins mi-vésiculés; c'est aussi le cas de Mills (1969) en Ecosse. Bulleid (1973) propose un déversement au stade $0^{+}$car il assure une meilleure dispersion des poissons.

Les conditions de transport (état physique de l'alevin au moment du déversement) peuvent également intervenir expliquant en partie les plus faibles survies des juvéniles en automne 1975.

\section{- la densité d'alevinage}

Dans cette étude, il n'apparaît pas de relation nette entre la densité au déversement et le taux de survie automnale. Pourtant une diminution de la densité d'alevinage semble entraîner des survies et des densités ou égales (Mills 1964, 1969) ou supérieures aussi bien au stade $0^{+}$(Anonyme $1973 \mathrm{a}$, Egglishaw et Shackley 1973) qu'à celui de $1^{+}$an (Jones 1970, Elson 1975). Des densités initiales trop élevées peuvent abaisser le taux de survie compte tenu d'un changement possible de compétition qui, de spatiale, deviendrait alimentaire dans les ruisseaux (Bulleid 1973) et d'une augmentation parallèle de la densité et de l'agressivité (Fenderson et Carenter 1971). Ce mécanisme provoquerait une mortalité (prise au sens large) par suite d'une dévalaison dans les mois suivant le déversement (Mills 1964 et 1969, Mortensen 


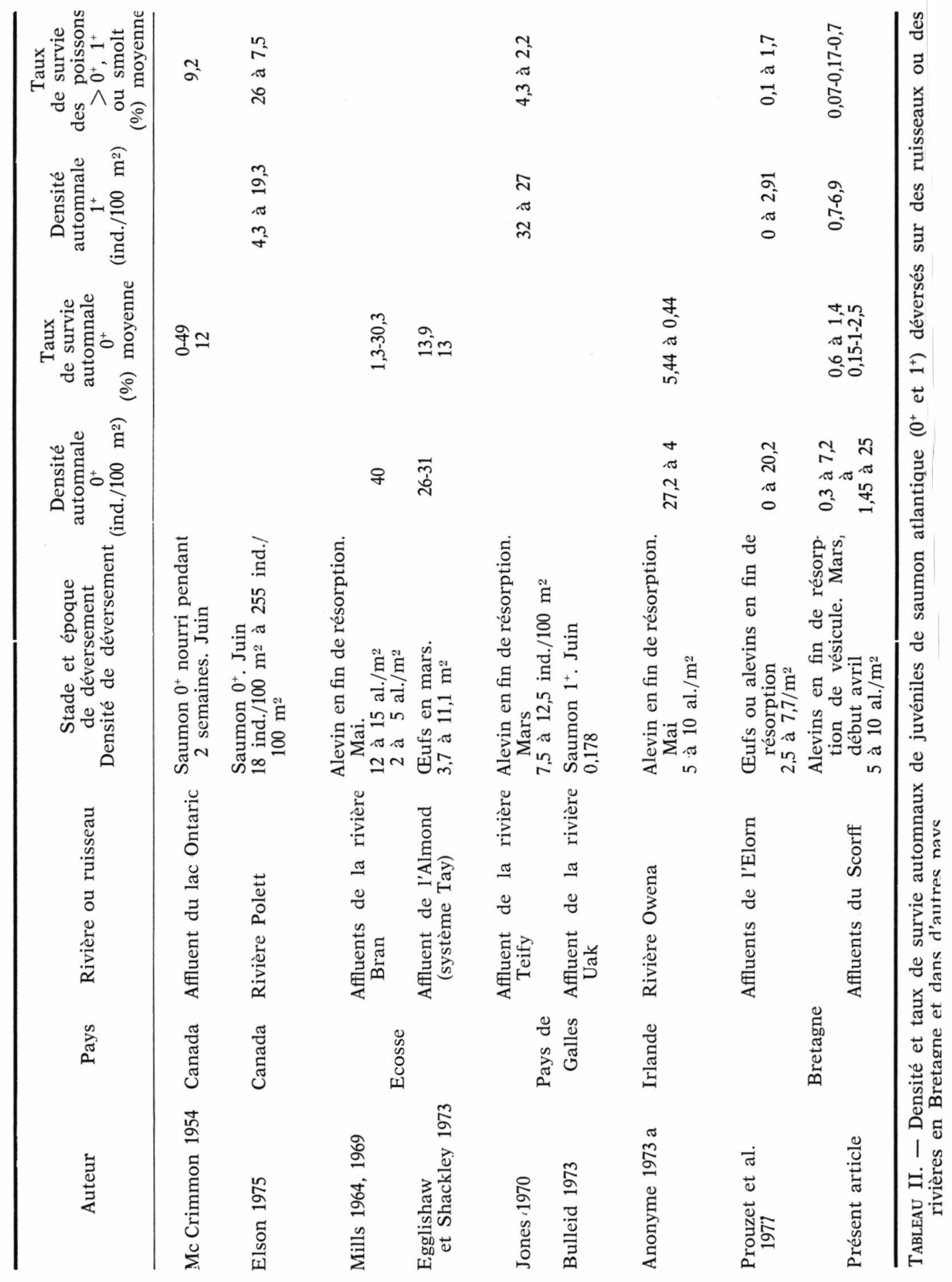


1977 sur la truite). Cette constatation a été faite sur le ruisseau de Kernec sans qu'ait pu être estimée l'amplitude de cette dévalaison. Ensuite tous ces auteurs s'accordent pour reconnaître une stabilité des effectifs dès le début de l'été, ce qui est constaté dans les quatre affluents du Scorff.

- le milieu physique

Les meilleurs résultats sont obtenus les années où les alevins sont déversé dans des zones de radiers (courant rapide, faible profondeur, substrat grossier) entrecoupées d'autres plus profondes. Les zones de radiers sont les types d'habitats préférés des juvéniles de saumon $0^{+}$ dans la rivière (Champigneulle, 1978) et la succession de radiers et de profonds permettrait une optimisation des rendements en ruisseaux pépinières (Mundie 1974). Dans ses ruisseaux, Mc Crimmon (1954) constate que la survie des saumons $0^{+}$est d'autant plus élevée qu'il y a de zones de courant à substrat grossier sans sédiments fins permettant, entre autres, une protection contre la prédation.

La création de barrages augmente la population de juvéniles parce que le milieu est rendu plus favorable : nombre supérieur de caches et rétention estivale de l'eau. Ce type d'amélioration de l'habitat proposé par Sedgewick (1970) est pratiqué avec succès sur des ruisseaux à truite en Irlande (Anonyme, 1973 b). Ceci est également utilisé pour améliorer la survie et la croissance des saumons "landlocked" d'un ruisseau du Maine mais la régularisation du débit n'a qu'une influence partielle sur la population des $0^{+}$(Havey et Davis 1970, Havey 1974).

- l'enlèvement des populations présentes dans le ruisseau

Le résultat de telles opérations apparaît controversé. Pour Burnett (1968), la suppression de la population d'anguilles (deux espèces) sur un cours d'eau de Nouvelle-Zélande permet d'augmenter de 3 à 10 fois la densité de population de truites mais le taux de croissance et le coefficient de condition diminuent. Pour Mills $(1964,1969)$ et Jones (1970) après suppression des salmonides âgés d'un an et plus, la densité et le taux de survie des saumons augmentent alors que dans une rivière irlandaise, après la même opération, on constate peu ou pas d'influence (Anonyme 1973 a). Sur l'Elorn, Prouzet et al. (1977) observent un effet positif de l'enlèvement des truites sur la population de saumons déversés mais ce dernier peut être masqué par la compétition existant entre les juvéniles $0^{+}$et $1^{+}$. En population naturelle Egglishaw et Shackley (1977) constatent une diminution du nombre de saumons $0^{+}$en liaison avec la densité combinée des truites et des saumons plus âgés. Enfin pour Flick et Webster (1975) la suppression pendant 13 ans de toutes les espèces accompagnant l'Omble (Salvelinus fontinalis) n'aboutit qu'à un arrêt de la mobilité de ce dernier après 7 ans. 
Sur les ruisseaux étudiés l'effet positif de l'enlèvement de toutes les espèces présentes lors de l'automne précédant l'alevinage peut s'expliquer :

+ par une très forte diminution de la densité d'anguilles (20-39,5 ind./ $100 \mathrm{~m}^{2}$ à $2-5$ ind. $/ 100 \mathrm{~m}^{2}$ ) dont la population peut exercer une pression sur celles des salmonides (Baglinière 1979).

+ par une diminution d'espèces compétitrices du saumon $0^{+}$. Ce peut être le cas du Chabot au niveau alimentaire (Mann 1971, Jones 1975). C'est surtout celui de la truite $0^{+}$très nombreuse sur les affluents du Scorff (Euzenat et Fournel 1976, Baglinière 1979) au niveau spatial et alimentaire. La mise en place de grilles peut avoir retardé la fraie des géniteurs de la rivière et donc diminué le nombre d'alevins de truites présents lors du déversement.

\subsection{Croissance}

Les quelques données recueillies sur la croissance des saumons naturels en ruisseau montrent que cette dernière est identique (voire inférieure) à celle des poissons déversés dans ce milieu. Sur l'Elorn, Prouzet et al. (1977) obtiennent la même croissance et observent le même phénomène. Sur les affluents de l'Elorn comme sur ceux du Scorff, ces déversements de saumon de souche écossaise donnent une proportion importante de smolts d'un montrant ainsi l'importance de l'environnement dans l'adaptation d'une population à un nouveau milieu.

La taille des smolts produits reste faible $(116 \mathrm{~mm})$ comparée à celle observée sur le Scorff $(188,6 \mathrm{~mm})$ et sur l'Ellé $(183 \mathrm{~mm})$ (Baglinière 1976) à proportion d'âge égale.

\subsection{Déplacements}

\section{- à l'intérieur du ruisseau}

Les déplacements des jeunes saumons à partir des secteurs ale. vinés sont observés sur d'autres ruisseaux mais ils sont plus faibles et leur direction plus aléatoire (Huntsmann 1945, Mc Crimmon 1954, Saunders et Gee 1964).

Les mouvements constatés à l'intérieur des ruisseaux peuvent avoir plusieurs causes. Les densités d'alevinage trop élevées pour des milieux à capacité biotique réduite peuvent provoquer un phénomène de dévalaison. Pourtant, en population naturelle, Egglishaw et Shackley (1977) considèrent que les saumons $0^{+}$ne migrent pas mais meurent sur place et que l'émigration commence à l'âge d'un an. En outre, en grossissant, le jeune saumon cherche un milieu plus adapté (Mc Crimmon 1954, Allen 1969, Symons et Heland 1978). Ces derniers auteurs, ainsi que Saunders et Gee (1964) et Symons (1974) observent des déplacements de saumons $0^{+}$des courants vers les profonds en 
automne. Ces divers phénomènes peuvent expliquer la diminution de densité des secteurs alevinés amont, corrélative à l'augmentation de celle de la zone aval sur le ruisseau de Kernec. Enfin, les mouvements peuvent être provoqués par des variations importantes du débit d'eau : crues (Huntsmann 1945) ou basses eaux (Saunders et Gee 1964).

\section{- à l'extérieur du ruisseau}

Lors de la dévalaison vers la rivière, les migrants du ruisseau de Kernec ne sont pas uniquement des saumons smolts mais également des saumons parrs. Cette descente de parrs en fin d'hiver et début du printemps est observée sur des ruisseaux écossais (Mills 1964, Egglishaw 1970, Anonyme 1969 et 1970) et canadiens (Mc Crimmon 1954). Il y aurait également une migration de saumons parrs en automne (Mc Crimmon 1954, Anonyme 1969 et 1970, Bulleid 1973). Sur le ruis. seau de Kernec, la capture d'un saumon parr en novembre 1976 permet de supposer que le phénomène existe également.

Ce possible mouvement de parrs à l'automne de même que la présence de smolts dans le ruisseau dès le mois de décembre laisse supposer que la période de descente étudiée du 11 janvier au 2 mai n'est pas entièrement couverte. Ainsi l'évaluation de la production en migrants reste biaisée. Mais cette descente des juvéniles vers la rivière montre que la production en migrants reste faible. Le pourcentage moyen de survie de l'alevin au smolt reste très inférieur $(3,8 \%)$ à celui observé (3\%) par Mc Crimmon (1954). Le taux de participation à la production totale de migrants dans la rivière reste faible (environ $1 \%$ ) en raison peut-être de la technique utilisée, mais surtout de la faible taille de cet affluent fortement colonisé par les juvéniles de truite.

\section{6. - CONCLUSION}

Les résultats de cette étude montrent l'intervention de nombreux facteurs sur la densité et la survie de juvéniles de saumons déversés au stade de fin de résorption de vésicule en ruisseau. Ce sont: la densité d'alevinage, l'importance de l'habitat, la suppression des espè ces pisciaires présentes. Tous ces facteurs ont été testés ensemble. Aucun d'entre eux ne semble avoir une influence prépondérante. Leur action reste synergique mais il apparaît cependant que :

- il est difficile de donner des densités d'alevinage correctes car leurs valeurs seront fonction de la capacité biotique du milieu;

- la suppression des populations pisciaires présentes ne sera favorable que si elle concerne des espèces à forte densité (Anguille) et/ou compétitrices (Truite $0^{+}$) ;

- l'amélioration du milieu (diversification du milieu, construction de barrages) peut augmenter sensiblement la production. 
Le rendement en migrants reste faible sans que pour autant que soit mise en cause la technique d'élevage extensif utilisée. Il contribue ainsi très peu à la production de la rivière. Une telle technique de production, compte tenu du milieu utilisé (ruisseaux à truitelles d'âge $0^{+}$et $1^{+}$) et de l'importance de la production en milieu naturel (tenir. compte des retours) peut reposer le principe de sa généralisation à des fins d'augmentation de production.

Il reste à comparer sur un même ruisseau les résultats obtenus dans cette étude avec ceux provenant d'une fraie naturelle (elle a lieu depuis déjà deux ans sur le ruisseau de Kernec) pour cerner l'impact d'une telle technique de production.

\section{TRAVAUX CITÉS}

ALLEN (K. R.). 1969. - Limitations on production in salmonid populations in streams. The University of British Columbia Institute of Fisherics. H. R. Macmillan Lectures in Fisheries, Symposium on Salmon and Trout in streams, 1968 , p. $3-18$.

Anonyme. 1969. - Report of the Freshwater Fisheries Laboratory, 1969. Pitlochry Department of Agriculture and Fisheries for Scotland. From Directorate of fisheries research, 106-151.

Anonyme. 1970. - Report of the Freshwater Fisheries Laboratory, 1970. Pitlochry Department of Agriculture and Fisheries for Scotland. From Directorate of fisheries research, p. 97-139.

Anonyme. 1973 a. - Inland Fisheries Bulletin Stationery office: Dublin, $n^{\circ} 10$ $37 \mathrm{pp}$.

Anonyme, 1973 b. - Annual Report 1972/73. The Inland Fisheries Trust incor. porated. Dublin, 1 vol., 79 pp.

Baglinière (J.L.). 1976. - Les populations de Saumon atlantique en Bretagne et єn Basse-Normandie. I. Caractéristiques des smolts sur la rivière Ellé. Ann. Hydrobiol., 7, 141-158.

BAGLINIERE (J.L.). 1979. - Les populations pisciaires en place sur une rivière à salmonides de Bretagne-Sud, le Scorff. Cibium, 7 : 53-74.

BurnetT (1968). in Mills (D. H.). 1970.

BulleID (H. J.). 1973. - The dispersion of hatchery reared atlantic salmon (Salmo salar) stocked into a fish less stream. Int. Atl. Salm. Found. Spec. Publi. Ser., 4, 169-179.

Champigneulle (A.). 1978. - Caractéristiques de l'habitat piscicole et de la population de juvéniles sauvages de saumon atlantique (Salmo salar L.) sur le cours principal du Scorff (Morbihan). Thèse 3e cycle: Biol. anim. Fac. Sci. Univ. Rennes, 1 vol., 92 pp.

Egglishaw (H. J.). 1970. - Production of salmon and trout in a strcam in Scotland. J. Fish. Biol., 2, 117-136.

Egglishaw (H. J.) et Shackley (P. E.). 1973. - A Experiment of faster growth of salmon Salmo salar L. in a Scottish Stream. J. Fish. Biol., 5, 197-204.

EgGlishaw (H. J.) et SHackley (P. E.). 1977. - Growth, survival and production of juvenile salmon and trout in a Scottish stream, 1966-75. J. Fish. Biol. 11, 647672.

Elson (P.F.). 1962. - Predator-prey relationships fish eating birds and atlantic salmon: Bull. Fish Res. Board Can., 133, 87 p. 
Elson (P. F.). 1975. - Atlantic salmon rivers, smolt production and optimal spawning: an overview of natural production. Int. Atl. Salm. Found Spec. Publ. Ser., 6, 96-119.

Euzenat (G.) et Fournel (F.). 1976. - Recherches sur la truite commune (Salmo trutta L.) dans une rivière de Bretagne, le Scorff. I. Caractéristiques démographiques des populations de truite commune de la rivière Scorff et ses affluents. II. Premiers éléments d'une étude de dynamique de population de Truite commune. Thèse $3^{c}$ cycle Biol. anim. Fac. Sci. Univ. Rennes, 1 vol., $213 \mathrm{pp}$.

Fenderson (O. C.) et Carenter (M. R.). 1971. - Effects of crowding on the behaviour of juvenile hatchery and wild Landlocked atlantic Salmon (Salmo salar L.). Anim. Behav., 19, 313: 439-447.

FLick (W. A.) et Webster (D. A.). 1975. - Movement, growth and survival in a stream population of wild brook Trout (Salvelinus fontinalis) during a period of removal of non Trout species. J. Fish. Res. Board Can., 32, 8, 1359-1367.

Fontenelle (G.), Baglinitire (J. L.), Prouzet (P.), Harache (Y.) et Douaire (G.) (en prép.). - The Atlantic Salmon Populations in Britany and Low-Normandy - 4. Characte ristics of adult salmon.

HAVEY (K. A.). 1974. - Effects of regulated flows on standing crops of juvenile salmon and other Fishes at Barrows Stream, Maine. Trans. Am. Fish. Soc., $103,1,1-9$.

Havey (K. A.) et Davis (R. M.). 1970. - Factors influencing standing crops and survival of juvenile salmon at Barrows stream, Maine. Trans. Am. Fish. Soc., 99, 297-311.

Huntsmann (A. G.). - Migration of salmon parr. J. Fish. Res. Board Can., 6, $399-402$.

JoNEs (A. N.). 1970. - A study of salmonid populations of ther river Teify and tributaries near Tregaron. J. Fish. Biol. 2, 183-197.

JoNES (A. N.). 1975. - A preliminary study of fish segregation in salmons spawning stream. J. Fish. Biol., 7, 95-104.

MANN (R. H. K.). 1971. - The populations, growth and production of fish in four small streams in Southern England. J. Anim. Ecol., 40, 155-190.

Mc Crimmon (H. R.). 1954. - Stream studies on planted atlantic salmon. J. Fish. Res. Bd. Canada, 11 (4), 362-403.

Mills (D. H.). 1964. - The Ecology of the young stages of the atlantic salmon in the river Bran Ross-Shire. Freshwat. Salm. Fish. Res., 32, 58 p.

MiLls (D. H.). 1969. - The survival of hatchery-reared salmon fry in some scottish streams. Freshwat. Salm. Fish. Res., 39, 12 pp.

Mills (D. H.). 1970. - Preliminary observations in fish populations in some Twced tributaries. Annual Report to the River Tweed commissionners, $4 \mathrm{pp}$.

Mortensen (E.). 1977. - Population, survival, growth and production of trout Salmo trutta in a small danish-stream. Oikos, 28, 9-15.

Mundie (J. H.). 1974. Optimisation of the salmonid nursery stream. J. Fish. Res. Board. Can., 31, 1827-1837.

Prouzet (P.), Harache (Y.), Danel (P.) et Brannnelec (J.). 1977. - Extensive production of atlantic salmon parrs (Salmo salar L.) in four nursery streams of northern Brittany. ICES Anadromous and Catadromous Fish. Committee. C.M. 1977/M : 18, 25 pp.

SAunders (R. L.), GeE (J.H.). 1964. - Movements of young atlantic salmon in a small stream. J. Fish. Res. Board. Can., 21, 27-36.

SEDGEwick (S.). 1970. - Management of salmon rivers. The two lakes Second Fishery Management Training Course Report, 1970, p. 149-153. 
Symons (P. E. K.). 1974. - Territorial behaviour of juvenille atlantic salmon reduces predation by brooktrout. Can. J. Zool, 52, 677-679.

Symons (P. E. K.) et Heland (M.). 1978. - Stream habitats and behavioural interac. tions of under yearling and yearling Atlantic Salmon (Salmo salar). J. Fish. Res., Board Can., 35, 175-183.

Février 1980. 